\title{
Stable Clathrin: Uncoating Protein (hsc70) Complexes in Intact Neurons and Their Axonal Transport
}

\author{
Mark M. Black, ${ }^{1}$ Matthew H. Chestnut, ${ }^{2}$ Irene T. Pleasure, ${ }^{2,3}$ and James H. Keen ${ }^{2,3}$ \\ 1 Department of Anatomy and Cell Biology, ${ }^{2}$ Fels Institute for Cancer Research and Molecular Biology, and ${ }^{3}$ Department of \\ Biochemistry, Temple University School of Medicine, Philadelphia, Pennsylvania 19140
}

We have studied the organization of clathrin during its transport in axons. Using immunoprecipitation techniques we have confirmed earlier findings that clathrin is transported as part of slow component $b$, but we also detect small amounts of clathrin in fast component. As fast component is known to correspond to the transport of membranous material, including coated vesicle membrane components, our findings suggest that some clathrin in axons undergoes transport in the form of coated membranes and that a portion of the clathrin delivered to axons and axon terminals arrives by way of fast component. The organizational form of clathrin in slow component $b(\mathrm{SCb})$ was examined in more detail, as it is thought to represent a non-membrane-associated species, is relatively long-lived, and at any instant represents the major transport species in axons. We used nondenaturing immunoprecipitation methods with stringent wash procedures to identify other $\mathbf{S C b}$ proteins that interact with clathrin. The immunoprecipitates contained major labeled bands that corresponded to clathrin heavy and light chains, along with a prominent 70-kDa band and several minor bands that ranged in apparent $M_{r}$ from 70,000 to 150,000 ; the 70 kDa band was shown to be the ATP-dependent uncoating protein by two-dimensional gel electrophoresis. A very similar profile of polypeptides was also immunoprecipitated from extracts of cultured neurons. The results from a variety of control immunoprecipitations, including the use of antisera preadsorbed with purified clathrin trimers or clathrin light chains, indicate that coprecipitation of clathrin and uncoating protein with the other $70,000-150,000-\mathrm{Da}$ polypeptides from $\mathrm{SCb}$ reflects specific interactions. Including exogenous uncoating protein in the lysis buffer had no detectable effect on the levels of endogenous uncoating protein recovered in the immunoprecipitates, indicating that complexes of clathrin, uncoating protein, and the other coimmunoprecipitating $\mathrm{SCb}$ proteins existed in the intact neurons prior to lysis. Finally, a specific and functional association is further supported by the release of uncoating protein, but not the other 70,000-150,000-Da polypeptides, from the immunoprecipitated complexes on the addition of ATP. Collectively, these

\footnotetext{
Received Sep. 14, 1990; revised Nov. 19, 1990; accepted Nov. 20, 1990.

We are grateful to Dr. Cliff Steer for the uncoating protein preparation used in the initial phase of this study. This work was supported by Grants NS-17681 (to M. M. B.) and GM-28526 (to J. H. K.) from the National Institutes of Health. Correspondence should be addressed to Dr. Mark M. Black, Department of Anatomy and Cell Biology, Temple Untversity School of Medicine, Philadelphia, PA 19140.

Copyright (C) 1991 Society for Neuroscience $0270-6474 / 91 / 111163-10 \$ 03.00 / 0$
}

observations provide the first direct evidence of interaction between clathrin and uncoating protein in intact cells, lend strong support to the concept that uncoating protein plays an intimate role in clathrin dynamics within cells, and reveal a family of 70,000-150,000-Da polypeptides that form a stable nonmembranous association with clathrin in intact cells.

Studies of clathrin organization in intact cells have revealed assembled and unassembled forms that correspond to a membrane-associated coat structure and to a nonmembranous, apparently soluble cytoplasmic form, respectively (Louvard et al., 1983, Brodsky, 1985; Goud et al., 1985). In the intact cell clathrin is believed to cycle repeatedly between these forms, as, for example, in the uncoating and reformation of coated pits and vesicles at the plasma membrane during the process of receptormediated endocytosis (Goldstein et al., 1985).

The assembly of clathrin in cells is thought to be regulated at least in part by one or more of the assembly proteins (APs) because of the potent coat assembly activity of APs under in vitro conditions (Keen, 1990a,b). This view is reinforced by the colocalization of the APs with assembled clathrin in situ (Robinson and Pearse, 1986; Robinson, 1987; Ahle et al., 1988). Because the clathrin lattice of coated membranes is quite stable under physiological conditions of $\mathrm{pH}$ and ionic strength, it has also been suggested that the uncoating of such membranes is an active process (Patzer et al., 1982; Rothman and Schmid, 1986). An ATP-dependent uncoating protein (UP) has been identified and extensive studies with purified proteins have demonstrated its ability to disassemble the clathrin lattice of coated vesicles under in vitro conditions (Schmid and Rothman, 1985a,b; Schmid et al., 1985; Rothman and Schmid, 1986). The UP is identical to one of the constitutively synthesized $70-\mathrm{kDa}$ heat shock "cognate" proteins, termed hsc70 (Ungewickell, 1985; Chappell et al., 1986). Genetic and biochemical studies have provided evidence that members of this family of proteins, and the related $78-\mathrm{kDa}$ homolog in the endoplasmic reticulum, are involved in protein unfolding and refolding events of both native and aberrant proteins (Gething et al., 1986; Chirico et al., 1988; Deshaies et al., 1988; Zimmermann et al., 1988; Hurtley et al., 1989; Pelham, 1989). These observations, the presence of substantial amounts of UP relative to clathrin in many cells (Schlossman et al., 1984; Davis and Bennett, 1985; Davis et al., 1986; Giebel et al., 1988; Perkins et al., 1990) and the quite different response of UP and clathrin to heat stress (Ungewickell, 1985), have led to questioning of the view that uncoating of clathrin-coated membranes is a major, or possibly even a significant, function of the UP/hsc70 in cells. In short, there has 
been no direct evidence of either transient or long-term interactions between clathrin and UP in intact cells.

Neurons provide a useful model system to characterize cytoplasmic forms of clathrin and UP. Neurons synthesize relatively high levels of clathrin, much of which is conveyed from the cell body into the axon and axon terminal by the process of axonal transport (Garner and Lasek, 1981). Proteins undergo axonal transport at discrete rates in axons (reviewed in Grafstein and Forman, 1980). Three major rate classes of axonal transport have been identified and termed fast component (FC), slow component b (SCb), and slow component a (SCa). FC has an average transport rate of $250-400 \mathrm{~mm} / \mathrm{d}$ and corresponds to the transport of membranous structures. $\mathrm{SCa}$ and $\mathrm{SCb}$ are transported much more slowly than FC and they represent the movement of distinct groups of cytoplasmic proteins. SCa has a transport rate of $0.2-1.5 \mathrm{~mm} / \mathrm{d}$ and corresponds primarily to the transport of microtubule and neurofilament proteins (Lasek et al., 1984). SCb has a transport rate of $2-5 \mathrm{~mm} / \mathrm{d}$. SCb contains hundreds of distinct proteins, including glycolytic enzymes, actin, myosin, calmodulin, synapsin, and clathrin (Willard, 1977; Black and Lasek, 1979; Brady and Lasek, 1981; Brady et al., 1981; Garner and Lasek, 1981; Baitinger and Willard, 1987; Oblinger et al., 1988); clathrin represents $\approx 1 \%$ of the total material of SCb (Garner and Lasek, 1981). As SCb does not contain detectable intrinsic membrane components, the transport form of SCb clathrin is most likely nonmembranous. In typical axons, which have lengths of millimeters to centimeters, it takes days to weeks for $\mathrm{SCb}$ clathrin to be transported from the cell body to the axon terminal, where it may function in the membrane recycling processes associated with neurotransmission (Heuser and Reese, 1973). Thus, an apparently nonmembranous transport form of clathrin is a relatively long-lived species in intact neurons, particularly in the axon.

In considering the nature of the transport form of SCb clathrin, it is noteworthy that $\mathrm{SCb}$ also contains two families of clathrin-binding proteins, the UP and the AP (Chestnut et al., 1986; de Waegh and Brady, 1989). Cotransport of these proteins provides circumstantial evidence for a long-term association of one or both of these proteins with clathrin during their transport in the axon but the significance of this observation is clearly limited by the enormous compositional complexity of SCb (Black and Lasek, 1980; Garner and Lasek, 1982). In the present studies, we have used nondenaturing immunoprecipitation in an attempt to isolate the transport form of SCb clathrin. Using an antibody specific for clathrin light chain $b$, we found that $a$ discrete subset of minor $\mathrm{SCb}$ proteins was indeed specifically immunoprecipitated with clathrin, and demonstrated that the major coprecipitating protein was the 70-kDa UP. Furthermore, a similar complex containing clathrin and other cellular polypeptides could also be isolated by immunoprecipitation from cultured neurons. These results provide the first definitive demonstration of a stable association of clathrin and UP in situ, and raise the possibility that clathrin, $\mathrm{UP}$, and other $\mathrm{SCb}$ proteins exist in a novel form that may be specialized, in part, for intracellular transport.

\section{Materials and Methods}

Protein preparations. Bovine brain clathrin was prepared by gel filtration with Superose 6B (Pharmacia Fine Chemicals, Piscataway, NJ) as described previously (Keen, 1987). Enriched preparations of clathrin light chains were also prepared from bovine and rat brains as follows. All steps were performed at $0-4^{\circ} \mathrm{C}$ unless otherwise noted. We homogenized $\approx 10 \mathrm{gm}$ of brain in 2 volumes of buffer $\mathrm{A}(0.1 \mathrm{~m}$ NaMES, pH $6.5,1.0$
mM EGTA, $0.5 \mathrm{mM} \mathrm{MgCl}, 0.02 \% \mathrm{Na}$ azide, $0.5 \%$ Trasylol, and $10 \mu \mathrm{g} /$ $\mathrm{ml}$ cach of lcupcptin, pepstatin and antipain) using three strokes of a motorized glass-Teflon homogenizer. The homogenate was centrifuged for $30 \mathrm{~min}$ at $14,500 \times \mathrm{g}$, and the supernate recentrifuged for $60 \mathrm{~min}$ at $81,000 \times g$ in a Beckman type 40 rotor. The high speed pellet was

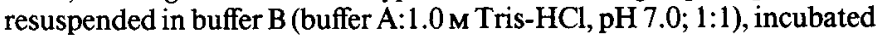
at room temperature for $10 \mathrm{~min}$, and then placed in a boiling water bath for $5 \mathrm{~min}$. The material was then centrifuged for $60 \mathrm{~min}$ at 81,000 $\times g$ to obtain a heat-stable supernate which contained predominantly clathrin light chains (Lisanti et al., 1982; Fig. 1). Initially, ATP-dependent uncoating protein was the generous gift of Dr. Cliff Steer and was subsequently prepared according to published procedures (Heuser and Steer, 1989). The purified UP used for these experiments was active as judged by its ability to uncoat reassembled coats in vitro (data not shown).

Radiolabeling axonally transported proteins. The present studies have examined SCb and FC for clathrin-UP complexes. Labeled proteins of $\mathrm{FC}$ or $\mathrm{SCb}$ were obtained from optic nerves and tracts of animals killed at $2.25-2.5 \mathrm{hr}$ or 6-7 d postinjection, respectively. Injections were performed on male CD rats weighing 350-550 gm (Charles River Laboratories, Wilmington, MA) anesthetized with $50 \mathrm{mg} / \mathrm{kg}$ Nembutal (Abbott Laboratories, North Chicago, IL) administered intraperitoneally. ${ }^{35} S$-methionine (Amersham Corp., Arlington Heights, IL), $0.5-1.5 \mathrm{mCi}$, was lyophilized and redissolved in $5 \mu \mathrm{l}$ of deionized water and then injected into the vitreous of the eye. For most experiments, both eyes of each rat were injected with isotope, and the optic nerves were either pooled or analyzed separately for clathrin by immunoprecipitation (see below). In addition, control experiments were carried out in which one eye from each rat was injected with ${ }^{35} S$ met and then the optic nerves from the injected and uninjected cycs were assayed for labeled clathrin by immunoprecipitation. In all of these latter experiments, the levels of labeled clathrin and other coimmunoprecipitating proteins in the material from the injected eye greatly exceeded that from the uninjected eye (see Fig. 3, compare lanes 2 and 4 and data not shown). Thus, local incorporation of ${ }^{35} \mathrm{Smet}$ into protein by non-neuronal cells in the optic nerve was not a significant factor in the experiments reported here.

Immunological procedures. A rabbit antisera to bovine brain clathrin trimers (Keen et al., 1981) was used in immunoblotting and immunoprecipitation analyses. For evaluation of clathrin heavy chain proteolysis, a monoclonal anti-bovine brain clathrin heavy chain antibody $\left(\mathrm{C}_{\mathrm{HC}} 5.9\right.$, Boehringer-Mannheim) was used in immunoblotting analysis. Immunoblotting was carried out as described previously (Peng et al., 1985). Immunoprecipitation was used to assay SCb and FC for clathrin. Optic axons containing labelcd SCb or FC were homogenized under denaturing or nondenaturing conditions as indicated. For denaturing conditions, the tissue was homogenized in $1 \%$ SDS containing protease inhibitors, boiled for $5 \mathrm{~min}$, clarified by centrifugation, and then diluted with 9 volumes of RIPA buffer [50 mM Tris- $\mathrm{HCl}, \mathrm{pH} 7.2,150 \mathrm{~mm}$ $\mathrm{NaCl}, 1 \%$ Triton $\mathrm{X}-100,0.1 \% \mathrm{SDS}$, protease inhibitors (the same cocktail as in Buffer A)]. For nondenaturing conditions, the tissue was homogenized in buffer $B$ or in RIPA buffer, and then centrifuged for 30 min at $100,000 \times g ; \approx 70 \%$ of labeled SCb proteins and $\approx 90 \%$ of the labeled FC proteins were solubilized under these nondenaturing conditions. The supernates were then preincubated for $30-60 \mathrm{~min}$ at $4^{\circ} \mathrm{C}$ on a rotator with protein A-Sepharose (Pharmacia Fine Chemicals, Piscataway, NJ) equilibrated in the appropriate buffer. The protein A-Sepharose was removed by centrifugation, the preadsorbed extract was divided into appropriate numbers of cqual-sized aliquots and incubated with either an immunoglobulin fraction of antiserum or nonimmune serum; for some experiments, antiserum was preadsorbed with clathrin trimers or clathrin light chains. Immunoglobulin fractions of antiserum and nonimmune serum were used at a dilution of 1:100 (vol vol). Immunoglobulin fractions of antiserum were preadsorbed with antigen by incubation for $60 \mathrm{~min}$ with $0.33 \mathrm{mg}$ clathrin trimers or $1 \mathrm{mg}$ clathrin light chains per milliliter of immunoglobulin fraction. The labeled samples were incubated with antibody for $30-60 \mathrm{~min}$ at $4^{\circ} \mathrm{C}$ and then incubated with $5 \mathrm{mg}$ protein A-Sepharose equilibrated in the appropriate buffer containing $2 \% \mathrm{BSA}$, for $\geq 2 \mathrm{hr}$ at $4^{\circ} \mathrm{C}$ on a rotator. Antibody-antigen complexes bound to the protein A-Sepharose were collected by centrifugation, washed twice with RIPA buffer, spun through a $1-\mathrm{ml}$ cushion of $0.5 \mathrm{M}$ sucrose $+2 \mathrm{M}$ urea in RIPA buffer, and then rinsed sequentially with RIPA $+2 \mathrm{M}$ urea, RIPA without Triton X-100 and SDS, and finally $10 \mathrm{~mm}$ phosphate buffer, $\mathrm{pH}$ 7.0. Proteins bound to the beads were then solubilized by boiling in electrophoresis sample buffer.

Studies of clathrin-UP complexes in cultured neurons. These analyses 


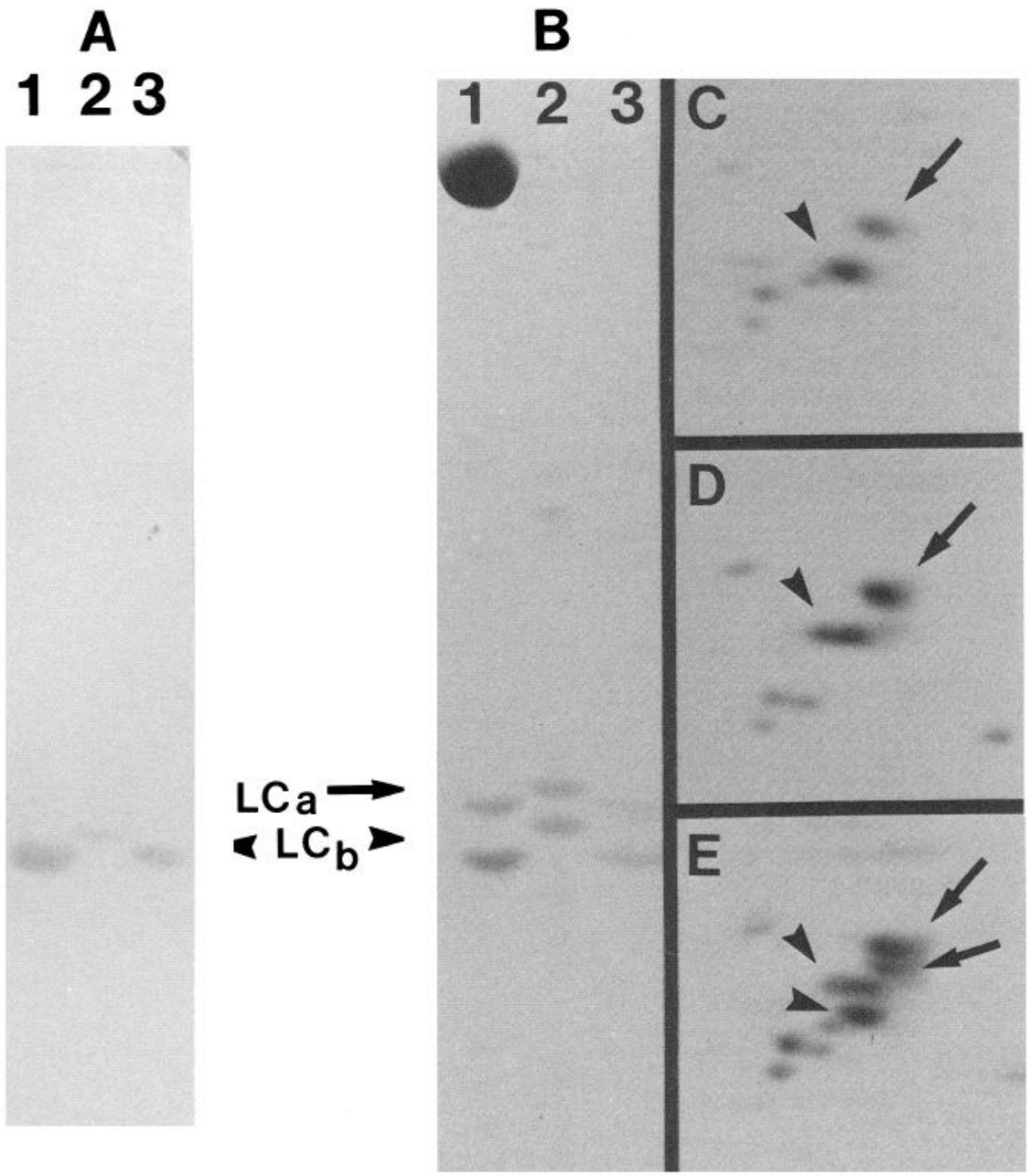

Figure 1. Immunological and biochemical analysis of brain clathrin light chains. Panel A, Immunoblotting analysis of clathrin trimers and purified light chains from calf and rat brain. Lanes 1, 3, Clathrin trimers and purified light chains, respectively, from calf brain. Lane 2, Clathrin light chains from rat brain. A single band is recognized in all three samples; the band in the calf brain samples comigrates precisely with authentic $\mathrm{LCb}$; the band in the rat brain sample migrates slightly slower than that from calf brain. Panel $B$, Coomassie blue-stained gel loaded identically to the blot shown in panel $A$. Panels $C-E$ Analysis of calf and rat brain light chains by $2-\mathrm{D}$ gel electrophoresis. Shown are reproductions of portions of the Coomassie blue-stained gels containing calf brain light chains (panel $C$ ), rat brain light chains (panel $D$ ), or mix of calf and rat brain light chains (panel $E$ ). In all panels, the arrows point to light chain a $(L C a)$ and the arrowheads point to light chain $\mathrm{b}(L C b)$.

used 10-14-d-old dissociated cultures of rat sympathetic neurons prepared and metabolically labeled as described previously (Peng et al., 1985). These cultures typically consist of $>95 \%$ neurons. For most experiments, cultured neurons were lysed by homogenization in buffer C (RIPA without SDS), and the resulting extracts were clarified by centrifugation for $10 \mathrm{~min}$ at $245,070 g_{\max }$ at $2^{\circ} \mathrm{C}$; variations on the lysis procedure are discussed in the Results section. The supernates were then assayed for clathrin by immunoprecipitation as described above, except that buffer $\mathrm{C}$ was used in place of RIPA buffer.

Gel electrophoresis. One-dimensional gel electrophoresis in SDS was as described previously (Keen, 1987). Two-dimensional isoelectric focusing $\times$ SDS gel electrophoresis was as described (Keen and Black, 1986) using an ampholine mixture of $0.5 \% \mathrm{pH} 3.5-10$ and $1 \%$ each of pH 4-6 and pH 5-6. Gels were stained with Coomassie brilliant blue. Labeled proteins were visualized by fluorography using either Enhance (NEN, Boston, MA) or PPO/DMSO (Bonner and Laskey, 1974); processed gels were incubated with preflashed XAR- 5 film (Eastman Kodak Co., Rochester, NY) at $-70^{\circ} \mathrm{C}$. Quantification of radioactivity in selected bands was performed by excising the bands from the gels, dissolving the gel pieces in $\mathrm{H}_{2} \mathrm{O}_{2}$, and then measuring the radioactivity by scintillation counting.

\section{Results}

\section{Clathrin and uncoating protein of SCb exist as a stable complex}

If clathrin and uncoating protein of $\mathrm{SCb}$ exist as a stable complex, then it should be possible to coimmunoprecipitate both proteins with a monospecific antibody to clathrin under nondenaturing conditions. We have tested this prediction using a polyclonal antibody prepared against gel filtration-purified bovine brain clathrin, which consists of a heavy chain plus two light chains designated $\mathrm{LCa}$ and $\mathrm{LCb}$. On immunoblotting of bovine brain clathrin trimers or partially purified clathrin light chains from bovine brain or rat brain, the antibody specifically recognized LCb (Fig. 1, panels $A, B$ ); a single band was also observed on immunoblotting of whole rat brain SDS extracts (data not shown). We note that the clathrin light chain bands from rat brain were slightly higher in apparent $M_{r}$ than their counterparts from bovine brain (Fig. 1, panels $B-E$ ).

The antibody can immunoprecipitate labeled clathrin from nerve extracts containing labeled SCb proteins. Previous studies have shown that $\mathrm{SCb}$ contains both clathrin heavy chain (Garner and Lasek, 1981) and light chains (Gower and Tytell, 1987) and we have confirmed the presence of $\mathrm{LCa}$ and $\mathrm{LCb}$ in $\mathrm{SCb}$ by $2-\mathrm{D}$ gel electrophoresis (Fig. 2B). When optic nerve containing labeled $\mathrm{SCb}$ proteins was dissolved in SDS, which fully dissociates and denatures clathrin trimers, and then assayed for clathrin by immunoprecipitation, a single labeled band was precipitated that comigrated with rat brain LCb in 1-D gels (Fig. 2, lane 2) and in 2-D gels (data not shown). The presence of this labeled material in the immunoprecipitates could be blocked completely by preadsorbing the antibody with clathrin trimers (Fig. 2, lane 1). These findings further establish the specificity of this antibody and document that it can be used in axonal transport studies. 


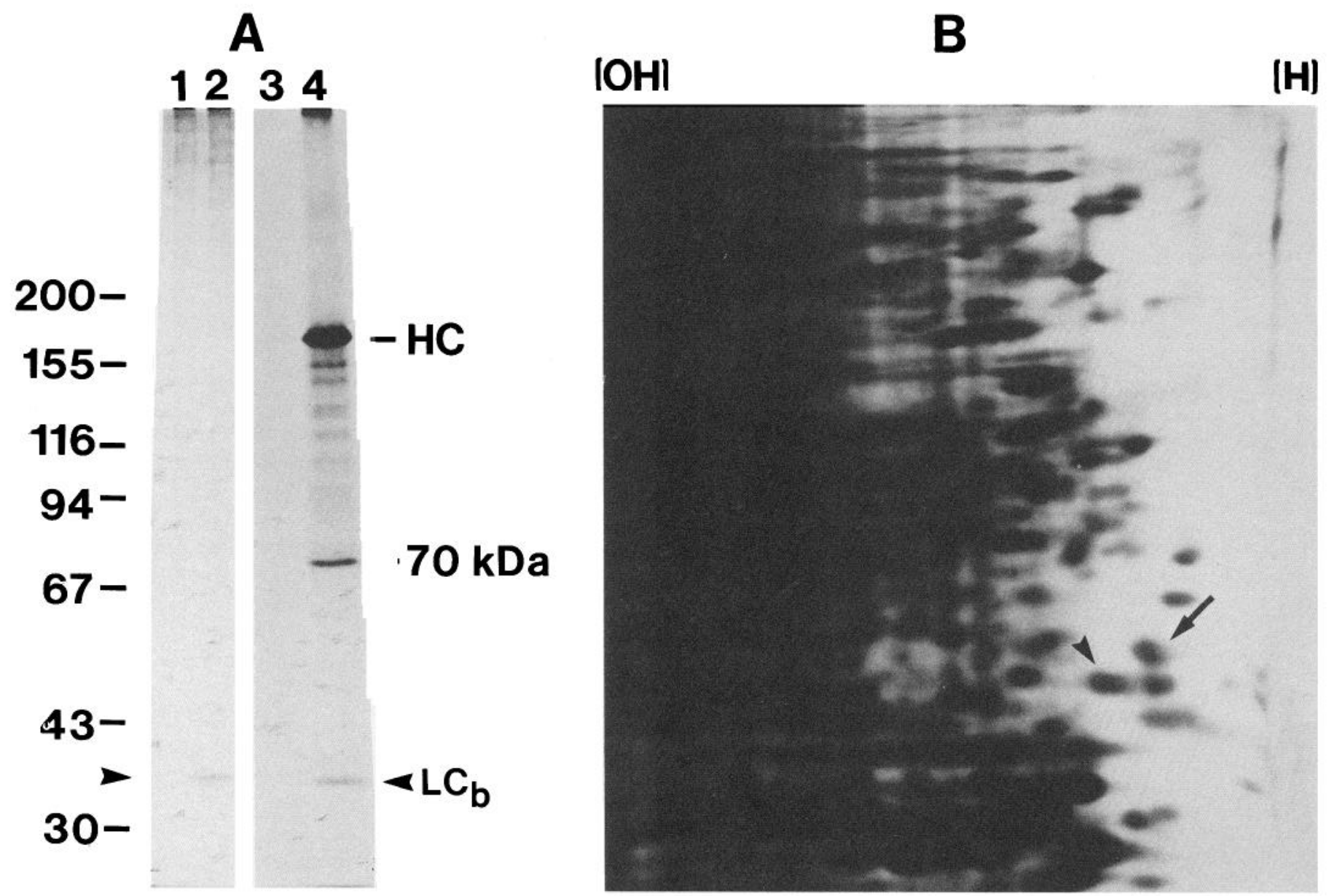

Figure 2. Immunoprecipitation of clathrin from SCb. Panel A, Immunoprecipitates from optic axons containing labeled SCb proteins that were dissolved in 1\% SDS (lanes 1,2) or RIPA buffer (lanes 3, 4). The precipitates in lanes 1 and 3 were obtained with antiserum preabsorbed with clathrin trimers, whereas those in lanes 2 and 4 were obtained with unabsorbed antiserum. In the sample dissolved in SDS, a single band was precipitated by the unabsorbed antisera but not by the preadsorbed antisera, and this band comigrated with rat LCb. In the sample dissolved in RIPA buffer, clathrin heavy and light chains plus several nonclathrin bands were precipitated by the unabsorbed antisera but not by the preadsorbed antisera. The numbers $(\times 1000)$ to the left of panel A indicate molecular weight. The positions of clathrin heavy chain $(H C)$, clathrin light chain $\mathrm{b}(\mathrm{LC} b ;$ arrowheads $)$ and the major $70-\mathrm{kDa}$ coimmunoprecipitating polypeptide are indicated to the right of panel A. Panel $B$, Optic axons containing labeled SCb proteins were dissolved directly in SDS and analyzed by 2-D isoelectric-focusing $\times$ SDS gel electrophoresis and fluorography. Unlabeled rat brain light chains were added to the sample as internal markers. The arrow and arrowhead indicate the positions of the carrier brain LCa (arrow) and LCb (arrowhead), respectively. Labeled proteins of SCb comigrate precisely with carrier rat brain LCa and LCb.

We used nondenaturing immunoprecipitation to look for stable interactions between clathrin and uncoating protein during axonal transport. Optic nerves containing labeled $\mathrm{SCb}$ proteins were extracted with either RIPA buffer or buffer B and then probed for clathrin-uncoating protein complexes by immunoprecipitation. Clathrin trimers remain intact under these solution conditions and can be immunoprecipitated by our anti$\mathrm{LCb}$ antibody. The most prominent band in these immunoprecipitates of $\mathrm{SCb}$ is the clathrin heavy chain, with an apparent $M$, of $180 \mathrm{~K}$, and bands corresponding to $\mathrm{LCa}$ and $\mathrm{LCb}$ are also present (Fig. $2 A$, lane 4 and, in a considerably heavier exposure from a replicate experiment, Fig. 3, lane 6). In addition, several other bands were present in the immunoprecipitates. The most prominent of these bands had an apparent $M$, of 70,000 with most of the other nonclathrin bands having apparent $M$, ranging from 70,000 to 150,000 (Fig. $2 A$, lane 4 and Fig. 3, lane 6). The same profile of bands was reproducibly immunoprecipitated in replicate experiments and was observed in extracts of nerve prepared in RIPA buffer or in buffer B.
The possibility that these $70,000-150,000-\mathrm{Da}$ polypeptides represent partially degraded clathrin heavy chains immunoprecipitated by the antibody is unlikely for several reasons. First, the antibodies responsible for immunoprecipitation are directed exclusively toward the clathrin light chains (see above). As these are much more proteolytically sensitive than the clathrin heavy chain (Ungewickell and Branton, 1981), complexes with degraded heavy chain would be expected to be devoid of light chain and not recognized by the antibody. Second, the diversity of the 70,000-150,000-Da polypeptides observed in the immunoprecipitates does not correspond to the specific and limited clathrin heavy chain fragments of 100,000-115,000-Da that are generated by a variety of different proteases in vitro (Kirchhausen and Harrison, 1984). Finally, as an indistinguishable pattern of 70,000-150,000-Da polypeptides has been observed in immunoprecipitates of cultured rat sympathetic neurons (see below), we directly lysed these cells in RIPA buffer and used an anti-clathrin heavy chain antibody to probe for heavy chain fragments by immunoblotting. In addition to intact heavy chain, 
minor species in the 100,000-115,000 Da range were observed, consistent with the pattern expected from in vitro proteolysis experiments, but unlike the pattern of the immunoprecipitates obtained with our anti-LCb antibody. Furthermore, incubation of the RIPA lysate for $6 \mathrm{hr}$ to mimic the immunoprecipitation protocol had no detectable effect on the relative levels of intact heavy chain and heavy chain fragments (data not shown), arguing against significant proteolysis in the sample after cell lysis. These observations indicate that proteolyzed clathrin heavy chain does not account for the 70,000-150,000 Da polypeptides observed in our nondenaturing immunoprecipitates.

The presence of nonclathrin bands in the immunoprecipitates obtained with the anti-LCb antibody could reflect nonspecific processes such as adsorption or trapping, the presence of contaminating antibodies, or specific interactions between the clathrin and nonclathrin proteins that survive immunoprecipitation. The possibility that the nonclathrin proteins are nonspecifically precipitated is unlikely for two reasons. First, a very stringent wash procedure, which included washing with RIPA buffer supplemented with $2 \mathrm{~m}$ urea (see Materials and Methods), was used in the present experiments. Second, if the presence of the nonclathrin proteins in the immunoprecipitates was due to nonspecific processes, then these proteins should for the most part correspond to major components of $\mathrm{SCb}$. This expectation was not supported by the data. The most heavily labeled $\mathrm{SCb}$ proteins range in apparent $M_{r}$ from $\approx 30,000$ to $\approx 70,000$ (Black and Lasek, 1980; Garner and Lasek, 1982; M. M. Black, M. H. Chestnut, I. T. Pleasure, and J. H. Keen, unpublished observations), whereas the most prominent $\mathrm{SCb}$ polypeptides that coimmunoprecipitate with clathrin range in apparent $M_{r}$ from 70,000 to 150,000 (Fig. $2 A$, lane 4 and Fig. 3, lane 6). These observations indicate that the coimmunoprecipitating proteins are not major components of $\mathrm{SCb}$ and that immunoprecipitation resulted in selective enrichment of a subset of minor $\mathrm{SCb}$ proteins.

To evaluate directly the contribution of nonspecific processes to the results, we have carried out immunoprecipitation analyses with antibody preadsorbed with purified unlabeled clathrin. Preadsorption should have minimal effect on nonspecific precipitation because immune complexes of antibody + unlabeled clathrin will be present and precipitated. These complexes should be equivalent to the antibody + labeled clathrin precipitated with nonadsorbed antibody with respect to trapping and nonspecific adsorption of $\mathrm{SCb}$ proteins. In addition, preadsorption experiments using purified clathrin provide stringent tests for the presence of contaminating antibodies that could be present in our anti-LCb antisera. Preadsorption of the anti- $\mathrm{LCb}$ antibody with either purified clathrin trimers or isolated clathrin light chains blocked not only the immunoprecipitation of labeled clathrin but also the $70-\mathrm{kDa}$ band and most of the other coprecipitating bands (Fig. $2 A$, lane 3 and Fig. 3, lane 5). Similar results were also obtained in other control experiments that used preimmune or various nonimmune antisera. These results provide direct evidence that nonspecific processes and contaminating antibodies are not responsible for the coimmunoprecipitation of nonclathrin proteins with clathrin under the nondenaturing conditions used in these experiments. Thus, we conclude that clathrin heavy and light chains, the $70-\mathrm{kDa}$ band, and a subset of nonclathrin proteins of $\mathrm{SCb}$ are specifically immunoprecipitated by the antibody, even though it is specific for LCb. The most likely explanation for the coprecipitation of these proteins with clathrin is that the nonclathrin proteins form

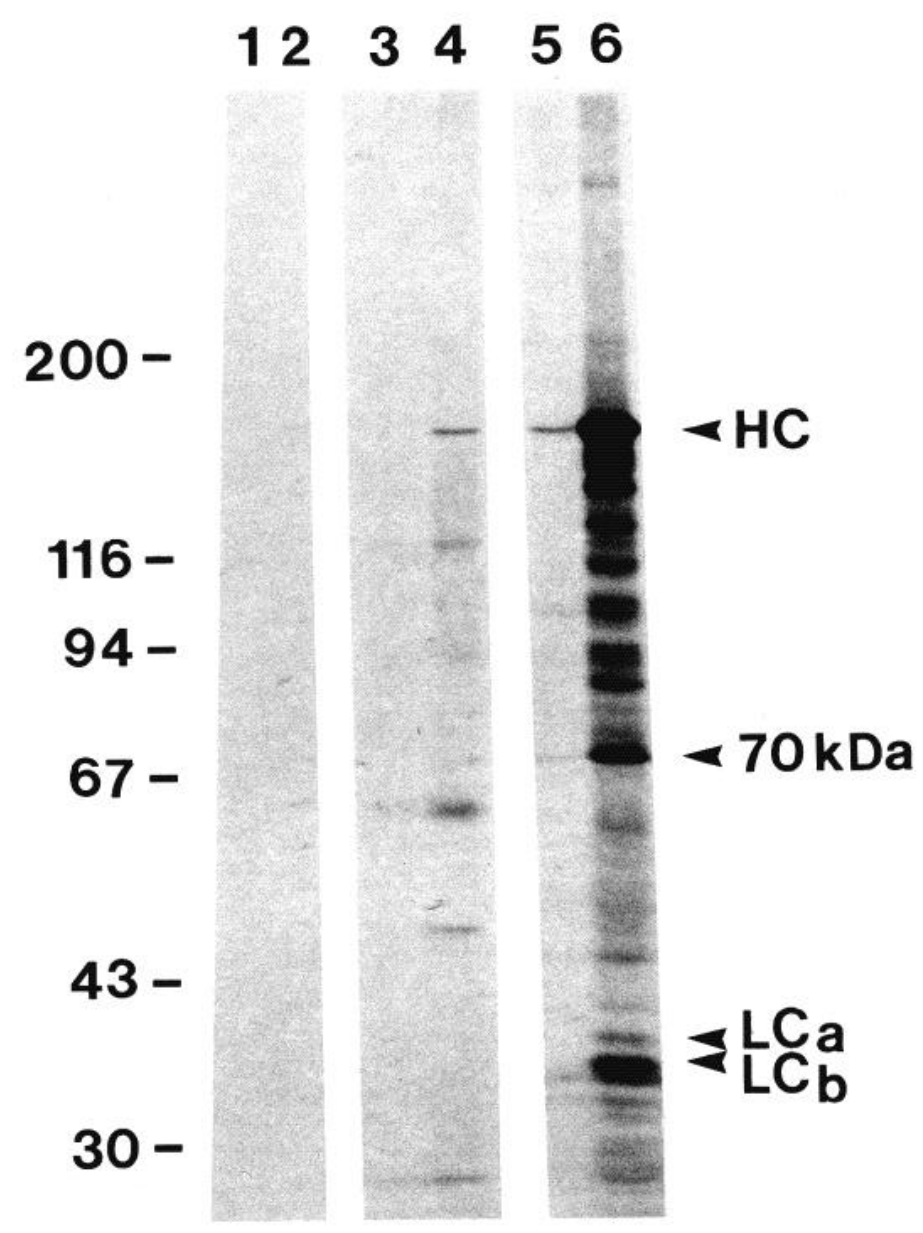

Figure 3. Identification of clathrin in FC and SCb. Optic nerves containing labeled proteins of either FC or SCb were extracted with RIPA buffer and assayed for clathrin by nondenaturing immunoprecipitation as described in Materials and Methods. Extracts of optic nerve containing equal $\mathrm{CPM}$ of either $\mathrm{FC}$ or $\mathrm{SCb}$ were also adjusted to contain equal nerve protein by adding appropriate volumes of identically prepared extracts from unlabeled nerve. The resulting samples, which thus contained the same amount of total radioactivity, the same amount of total nerve protein, and the same total amount of clathrin (i.e., labeled + unlabeled), were then challenged with the anti-clathrin light chain antibody. Lanes 1-4, Immunoprecipitates from the uninjected (lanes 1 and 2) or injected (lanes 3 and 4) optic nerve of a rat killed $2.5 \mathrm{hr}$ postinjection; the nerve from the injected eye contains labeled fast component proteins whereas the nerve from the uninjected eye serves as a control for local incorporation due to blood-borne delivery of ${ }^{35} \mathrm{~S}-\mathrm{L}$ Met. Lanes 5 and 6, Immunoprecipitates from the injected optic nerve of a rat killed $6 \mathrm{~d}$ postinjection and thus containing labeled $\mathrm{SCb}$ proteins. Immunoprecipitates obtained with unabsorbed antibody are shown in lanes 2,4 , and 6 , whereas those obtained with antibody preadsorbed with clathrin trimers are shown in lanes 1,3 , and 5 . The positions of molecular weight markers, clathrin heavy and light chains, and the 70$\mathrm{kDa}$ coimmunoprecipitating protein are indicated as in Figure 2.

one or more complexes with clathrin that are precipitated by the antibody. This view is strongly reinforced by the demonstration that the $70-\mathrm{kDa}$ coprecipitating protein is the UP, a known clathrin binding protein (see below).

\section{Clathrin is transported in fast component}

Previous studies using 2-D electrophoresis of axonal extracts containing labeled FC proteins have failed to detect clathrin in FC (Gower and Tytell, 1987). As this type of assay is relatively insensitive, we have used immunoprecipitation to reevaluate 
FC for the presence of clathrin (Fig. 3). Equal amounts of radioactive FC and $\mathrm{SCb}$ were challenged with the anti-LCb antibody (details of the procedure are noted in the figure legend). This experimental design reveals the proportion of $\mathrm{FC}$ and $\mathrm{SCb}$ comprised by clathrin but does not directly compare the total amounts of clathrin transported in each rate class.

Clathrin is detected in FC, though it comprises much less of this rate class than of $\mathrm{SCb}$ (Fig. 3, compare lanes 4 and 6). Nonetheless, these levels were considerably greater than those obtained from controls derived from uninjected eyes (see Fig. 3, lanes 2 and 4, and Materials and Methods), thereby confirming that clathrin is indeed transported in FC. The relative contribution of $\mathrm{FC}$ and $\mathrm{SCb}$ to the steady state levels of clathrin in the axon and axon terminal is a complex issue. The observations that $\mathbf{S C b}$ is labeled to a much greater extent than FC in pulselabeling experiments (Grafstein and Forman, 1980) and that clathrin comprises a much greater proportion of $\mathrm{SCb}$ than of FC (Fig. 3) suggests that much more clathrin is delivered to the axon by $\mathrm{SCb}$ than by FC. However, because the transport rate of $\mathrm{FC}$ is $\approx 100$-fold greater than $\mathrm{SCb}$, the role of $\mathrm{FC}$ in maintaining clathrin levels in the axon and axon terminal may be greater than that suggested solely by considering the amounts of clathrin in FC and $\mathrm{SCb}$.

The immunoprecipitates from $\mathrm{FC}$ also differed from those of $\mathrm{SCb}$ in another important respect. As seen earlier, the immunoprecipitate from $\mathrm{SCb}$ was very heterogeneous, consisting of clathrin heavy and light chains, and the 70-kDa uncoating protein, as well as many other bands. Nonclathrin proteins were also present in the immunoprecipitates from $\mathrm{FC}$, but unlike the situation for $\mathrm{SCb}$, most of these corresponded to major $\mathrm{FC}$ proteins (data not shown), thus raising the possibility that their presence in the immunoprecipitates was due to nonspecific processes. This prospect is reinforced by the finding that preadsorption of the antibody with clathrin trimers completely eliminated labeled clathrin from the precipitates but only partially reduced the levels of many of the nonclathrin proteins (Fig. 3, lanes 3 and 4 and data not shown). This contrasts with the result obtained with $\mathrm{SCb}$, in which preadsorption much more completely eliminated the nonclathrin proteins from the precipitates (Fig. 3, lanes 5 and 6).

\section{The 70-kDa band in immunoprecipitates from SCb obtained with the anti-LCb antibody is the clathrin uncoating protein}

Two observations suggested that the 70-kDa band of SCb that coprecipitated with clathrin was the UP. First, its apparent $M$, corresponds to that of the ATP-dependent UP (Schlossman et al., 1984). Second, during the course of these studies, transport of the UP in $\mathrm{SCb}$ was reported (de Waegh and Brady, 1989). To determine whether the $70-\mathrm{kDa}$ coprecipitating protein was indeed the UP, immunoprecipitates from nerve extracts containing labeled $\mathrm{SCb}$ proteins were obtained with the anti-LCb antibody and coelectrophoresed with authentic UP (generously provided by Dr. C. Steer) on 2-D gels. Autoradiographic exposures of the gels revealed a single labeled spot in the molecular weight range of $\approx 70,000$, and this spot comigrated precisely with the most acidic variant of the UP (Fig. 4). Labeled species comigrating with other, more basic, variants of the UP preparation were not detected, a point of interest as these species probably correspond to other variants within the $70-\mathrm{kDa} h s p /$ hsc family (Welch and Feramisco, 1985; Chappell et al., 1986; Ungewickell, 1985). We conclude that the $70-\mathrm{kDa}$ band that coimmunoprecipitates with SCb clathrin is indeed the UP.

\section{Clathrin-uncoating protein complexes in cultured neurons}

We have determined whether clathrin-UP complexes similar to those observed in $\mathrm{SCb}$ of rat retinal ganglion cell axons also exist in cultured sympathetic neurons. In addition to determining whether such complexes exist in other types of neurons, the culture system affords a much greater opportunity than the axonal transport system for detailed biochemical analyses of the nature of the clathrin-UP complex.

We first determined whether clathrin-UP complexes could be immunoprecipitated from extracts of cultured neurons. As shown in Figure 5, lane 1, clathrin heavy and light chains and a 70-kDa polypeptide were major components present in immunoprecipitates prepared in buffer $\mathrm{C}$, along with many other less prominent bands in the $M_{r}$ range of $70-150 \mathrm{~K}$. Identical results were also obtained when cultures were extracted with RIPA buffer (data not shown). The profile of bands present in the immunoprecipitates from extracts of cultured neurons was strikingly similar to that obtained in immunoprecipitates from $\mathrm{SCb}$ (see Fig. $2 A$, lane 4 or Fig. 3 , lane 6), suggesting that clathrincontaining complexes like those present in $\mathrm{SCb}$ of retinal ganglion cells are also present in cultured neurons. In this regard, various controls such as preadsorbing the antibody with purified clathrin trimers or using nonimmune serum blocked the appearance of labeled clathrin heavy and light chains, the $70-\mathrm{kDa}$ band and most of the lighter bands between 70 and $150 \mathrm{kDa}$ (data not shown). These observations confirm that these nonclathrin polypeptides are specifically coprecipitated with clathrin. The most prominent of these coprecipitating proteins has an apparent $M_{r}$ of 70,000 and corresponds to the UP.

To evaluate properly the biological significance of the coprecipitation of clathrin-UP complexes, it is necessary to determine whether the complex existed in situ or formed only artifactually following lysis of the neurons. If the complex formed postlysis, then unlabeled UP included in the lysis buffer should compete with the labeled UP and thereby reduce the levels of labeled UP in the resulting immunoprecipitates. We tested this prediction by including 1.2 or $6.0 \mu \mathrm{g}$ of purified brain UP in the lysis buffer. A typical culture yields $\approx 200 \mu \mathrm{g}$ of total protein. Assuming that UP represents $\approx 0.1 \%$ of total protein (Schlossman et al., 1984), then the levels of exogenous UP included in the lysis buffer are expected to exceed the endogenous UP by 6 - or 30-fold. As shown in Figure 5, including unlabeled UP in the lysis buffer had no detectable effect on the levels of labeled UP present in immunoprccipitates (comparc lancs 2 and 3 with lanc 1). Quantitative studies confirm the impression based on visual inspection; the ratio of DPM in clathrin to DPM in UP was 8.9 in control precipitates and 8.7 in precipitates prepared with a 30-fold excess of exogenous unlabeled UP in the lysis buffer.

In a final series of experiments, we asked whether the clathrinUP complex recovered by immunoprecipitation possessed a functional UP, as characterized by its ability to dissociate from clathrin in the presence of ATP (Flynn et al., 1989; Heuser and Steer, 1989). Immune complexes bound to protein A-Sepharose beads were prepared from soluble extracts of cultured neurons as described in Materials and Methods, and were then rinsed three times with buffer C. One aliquot of the clathrin-UP complexes bound to the beads was then incubated with ATP in the presence of an ATP-regenerating system for $30 \mathrm{~min}$ at $26^{\circ} \mathrm{C}$, while the other sample was incubated in parallel but without ATP. The beads were then sedimented through a cushion of buffer $C$ supplemented with $0.5 \mathrm{M}$ sucrose and $2 \mathrm{M}$ urea, and 

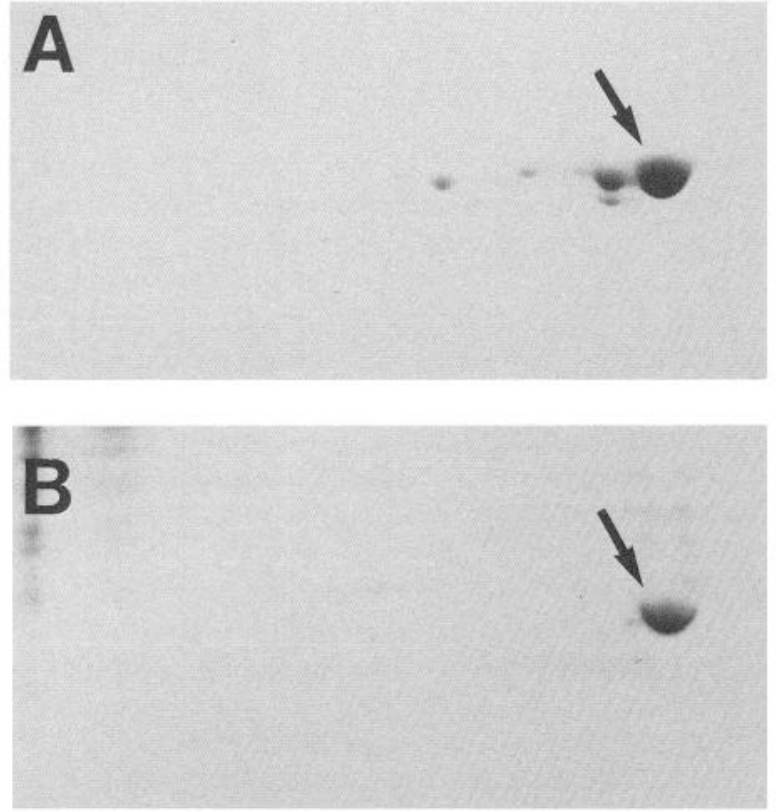

Figure 4. Clathrin uncoating ATPase is immunoprecipitated by anti$\mathrm{LCb}$ antiserum. Immunoprecipitates from optic axons containing labeled $\mathrm{SCb}$ proteins in RIPA buffer were analyzed by 2-D isoelectric focusing $\times$ SDS gel electrophoresis; purified UP was included in the sample as an internal marker. Panel $A$ shows a Coomassie blue-stained profile of the portion of the gel containing the UP. The basic end of the gel is to the left. The arrow in panels A and B identifies the position of the major Coomassie blue-stained spot. Panel $B$ shows a fluorograph of the same gel used to prepare panel A. Note that a labeled protein in the immunoprecipitates comigrates specifically with the most acidic variant of the UP preparation.

then further rinsed using the standard immunoprecipitation protocol. ATP treatment caused the selective release of the $70-\mathrm{kDa}$ UP (Fig. 5, compare lanes 4 and 5), demonstrating that the UP is present in a functionally active form in the immunoprecipitates, and by extension, in the intact cell. We also note that none of the other coprecipitating proteins in the immunoprecipitates were detectably affected by ATP treatment. Thus, we can also conclude that their presence in the immunoprecipitates is not due to their binding to the UP.

\section{Discussion}

The present studies provide the first demonstration that clathrin and UP form stable complexes in intact cells. The essential finding is that a polyclonal antibody specific for clathrin $\mathrm{LCb}$ coprecipitates clathrin in complex with UP under nondenaturing conditions. The specificity of the antibody was established by immunoblotting against purified clathrin and whole brain extracts as well as by immunoprecipitation out of heterogeneous nerve extracts prepared under denaturing conditions (Figs. 1 and 2 and data not shown). All of these assays showed that the antibody recognized a single band on SDS gels that corresponded to clathrin $\mathrm{LCb}$. The results from a variety of control immunoprecipitations indicate that the coprecipitation of clathrin and UP from labeled neuronal extracts reflects specific interactions (see Results). A specific and functional association is further supported by the ATP-dependent release of UP from the immunoprecipitated complexes (Fig. 5). These properties of the labeled rat neuronal proteins are entirely consistent with previous studies of clathrin-UP interactions using purified bo-

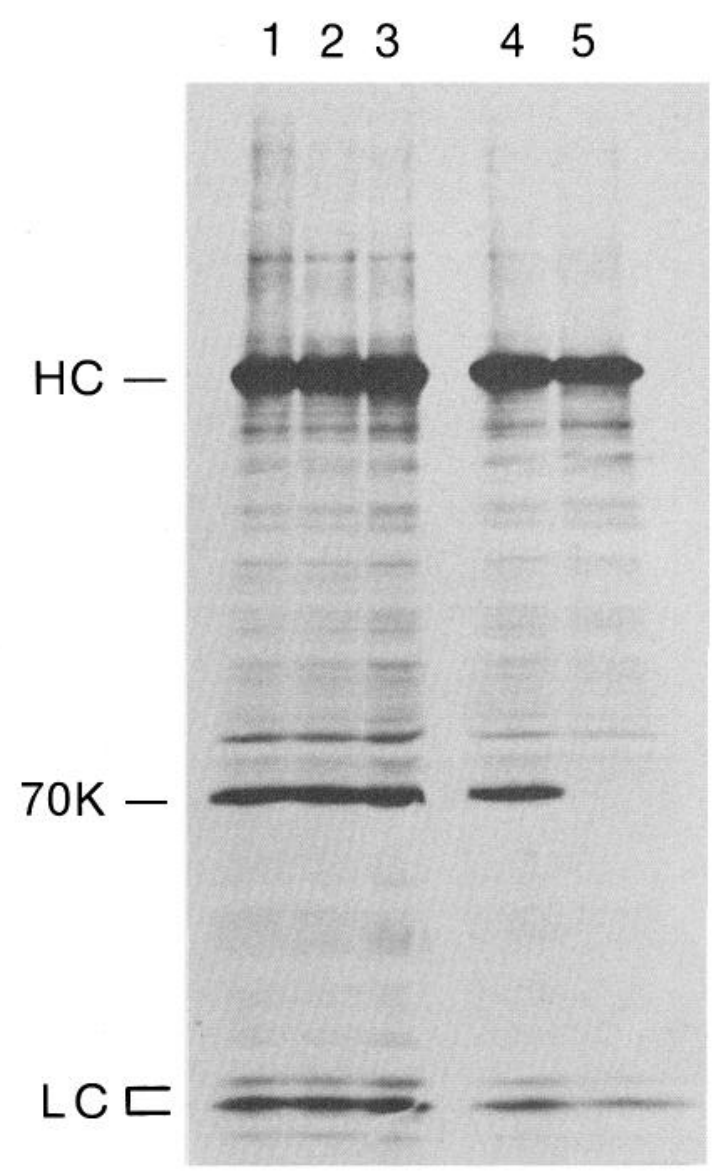

Figure 5. Immunoprecipitation of clathrin-UP complexes from extracts of cultured neurons. Lanes $1-3,{ }^{35} \mathrm{~S}-\mathrm{L}-\mathrm{Met}$ labeled cultures of sympathetic neurons were lysed in buffer $\mathrm{C}$ and immunoprecipitated with anti-clathrin antibodies (lane 1). Parallel cultures were lysed in the presence of $1.2 \mu \mathrm{g}$ or $6.0 \mu \mathrm{g}$ of UP/hsc70 (lanes 2 and 3 , respectively) and processed similarly (see Results for details). Lanes 4 and 5, Immune complexes bound to protein A-Sepharose, prepared from radiolabeled cells processed as in lane 1, were incubated at room temperature for 30 min in the presence of an ATP-regeneration system without ATP (lane 4) or with ATP (lane 5) and then washed and processed as described in Results. $H C$ and $L C$ refer to heavy and light chains of clathrin, respectively, while $70 \mathrm{~K}$ marks the UP/hsc70 band.

vine brain preparations (Braell et al., 1984; Schlossman et al., 1984; Schmid et al., 1985; Ungewickell, 1985; Chappell et al., 1986; Heuser and Steer, 1989).

Do clathrin-UP complexes normally exist in intact neurons? To answer this question, neurons labeled with ${ }^{35} \mathrm{~S}$-methionine were lysed in the presence or absence of up to a 30 -fold excess of unlabeled UP and then assayed for clathrin-UP complexes by nondenaturing immunoprecipitation. These experiments showed unambiguously that inclusion of exogenous UP in the lysis buffer had no detectable effect on the levels of endogenous (i.e., metabolically labeled) UP in the immunoprecipitates. The absence of measurable competition is strong evidence that complexes of clathrin and UP existed in the intact neurons prior to lysis. The complex as isolated in vitro is also evidently quite stable under the prevailing solution conditions, as the UP remains bound to clathrin throughout the immunoprecipitation procedure, which lasts several hours. Furthermore, addition of exogenous UP during the immunoprecipitation reaction does not appreciably displace cellular (i.e., labeled) UP from clathrin. 
Collectively, these observations provide the first demonstration of a relatively stable interaction between clathrin and UP in intact cells and lend strong support to the concept that UP plays an intimate role in clathrin dynamics in cells.

There is an apparent paradox in the premise that a clathrinUP complex exists as a stable entity within the cell, yet the UP can be readily released from the clathrin immunoprecipitate by physiological (or even lower) levels of ATP. One possible explanation for this apparent discrepancy is that the comparatively rapid ATP-dependent dissociation, which takes place on a time scale of minutes, has been shown to occur at very low protein concentrations (Flynn et al., 1989; Heuser and Steer, 1989), conditions that apply in the immunoprecipitation experiment reported here. In contrast, there is evidence suggesting that at higher protein concentrations (which presumably prevail in the intact cell) ATP-dependent release can be dramatically slower (Greene and Eisenberg, 1990), with an apparent half-time of many hours. Of course, other cellular factors may well affect the ATP-associated release of clathrin, which appears to be a complicated step in the overall uncoating reaction (Schmid and Rothman, 1985a,b; Schmid et al., 1985; Rothman and Schmid, 1986; Greene and Eisenberg, 1990). While resolution of this riddle will require further knowledge of the clathrin-UP interaction and the cellular environment, the data reported here clearly indicate that the clathrin-UP complex exists in the intact cell.

In addition to the UP, numerous other metabolically labeled polypeptides with apparent subunit $M_{r}$ in the range of 70-150 $\mathrm{K}$ also coimmunoprecipitated with clathrin, though with lower apparent stoichiometries. Although we have not studied these proteins as cxtcnsively as the UP, the'available data suggest that their presence in the immunoprecipitates is also due to specific interactions. This interpretation is supported by the absence of these bands in control immunoprecipitates (see Results), by the similarity in profiles obtained in immunoprecipitates from cultured neurons and $\mathrm{SCb}$ from optic nerves, and by the fact that most of the coimmunoprecipitating proteins are minor components of their respective starting materials. In view of the ability of the UP- $h s c 70$ to bind many unrelated peptides and presumably diverse proteins as well (Flynn et al., 1989; Rothman, 1989; Beckmann et al., 1990), it was anticipated that the presence of the heterogeneous group of coimmunoprecipitating proteins reflected interactions with UP. However, this expectation is not supported by the data, as treatment of the immune complexes with ATP resulted in the selective and nearly quantitative removal of UP without any discernible effect on the presence of the other immunoprecipitated proteins. We thus conclude that the presence of the coimmunoprecipitating proteins is not due to interactions with the UP but rather to binding directly or indirectly to clathrin. For simplicity, we refer to the material immunoprecipitated by anti-clathrin antibodies under nondenaturing conditions as "clathrin complexes," recognizing that the precise nature of these "complexes" remains to be defined.

It has been hypothesized that $\mathrm{SCb}$ corresponds to the transport of one or more multicomponent complexes (Garner and Lasek, 1981; Tytell et al., 1981). The evidence for this is indirect and is based in part on the observations that all proteins of $\mathrm{SCb}$ are transported together in the axon at a uniform rate and that the movement of the $\mathrm{SCb}$ proteins is absolutely distinct from the movement of the proteins transported in other rate components such as SCa and FC (for a complete discussion of this issue, see Garner and Lasek, 1982; Lasek et al., 1984). The present studies provide the first direct evidence in support of this hypothesis by showing that relatively stable complexes consisting of clathrin, UP, plus a discrete subset of other SCb proteins exist and can be isolated by immunoprecipitation from axonal extracts. The associations revealed by these immunoprecipitation experiments may represent only a subset of those occurring among the $\mathrm{SCb}$ proteins in situ. For example, the association of some $\mathrm{SCb}$ proteins with clathrin or other of the coprecipitating proteins may not have survived the dilution and/ or solution conditions associated with the immunoprecipitation protocol; this may explain why AP, which is in $\mathrm{SCb}$ (Chestnut et al., 1986), is not evident in our immunoprecipitates. Thus, the complexes identified by the present experiments may be part of one or more larger entities that comprise $\mathrm{SCb}$. Nondenaturing immunoprecipitation experiments similar to those performed here, but using antibodies to other $\mathrm{SCb}$ proteins and different solution conditions, should be useful to define better the organizational form of the $\mathrm{SCb}$ proteins.

What are the forms of axonally transported clathrin and of the complexed clathrin immunoprecipitated from neurons in culture? In considering these issues, it is relevant that clathrin is transported in both FC and SCb. Because the materials transported by these two rate classes are fundamentally different in their biochemical properties (Grafstein and Forman, 1980; Lasek et al., 1984), it is likely that the clathrin of SCb and FC are distinct in their intracellular organization. It is well documented that most and possibly all integral membrane components are delivered to the axon by $\mathrm{FC}$, and that these membrane components are transported in the-form of vesicles (Grafstein and Forman, 1980; Brady, 1984). We have shown that a portion of the axonally transported clathrin enters the axon with FC (Fig. 3). Because membrane components of coated vesicles are also transported in FC (Stone et al., 1984), and coated membrane profiles have been observed ultrastructurally in axons (Baas and Heidemann, 1986; Morre and Walter, 1982), it is likely that the clathrin of FC is transported as coated vesicles.

In contrast, the available evidence indicates that $\mathrm{SCb}$ clathrin is not transported in the form of coated membranes (see Garner and Lasek, 1981). First, integral membrane components have not been detected in SCb (Grafstein and Forman, 1980). Second, as shown here, a substantial fraction of $\mathrm{SCb}$ clathrin exists in a discrete complex with the ATP-dependent uncoating protein. Under in vitro conditions, clathrin is stabilized in a noncoat form when complexed with the uncoating protein (Schlossman et al., 1984; Rothman and Schmid, 1986). Finally, immunoelectron microscopic localization of clathrin in neurons reveals that while some is membrane associated, a substantial amount of clathrin is associated with nonmembranous components of the cytoplasm (Cheng et al., 1980; Lin et al., 1982).

What are the functions of the nonmembranous clathrin complexes transported in SCb? In considering this question, we note that coprecipitation with clathrin of major $70-\mathrm{kDa}$ as well as heterogeneous $70-150-\mathrm{kDa}$ polypeptides under nondenaturing conditions is not unique to experiments with neurons or to analyses with our polyclonal antibody. Strikingly similar patterns to those reported here were obtained by Brodsky (1985) in immunoprecipitation analyses of human lymphocyte cell lines using two different.monoclonal antibodies against clathrin heavy chain. At the-time it was not possible either to identify these bands or to be certain that the associations had actually preexisted in the intact cell, but the reasonable inference was made 
that they might represent clathrin-binding proteins (Brodsky, 1985). Recently, similar or identical complexes have also been observed in nondenaturing immunoprecipitates of labeled HeLa cells using monoclonal anti-hsp72,73 antibodies (Beckmann et al., 1990). These results then are consistent with the vicw that relatively stable complexes of clathrin, UP, and heterogeneous 70-150-kDa polypeptides exist in cells generally. If this is correct, then these complexes, rather than reflecting specializations unique to neurons, are likely to contribute to the structure and function of cells generally.

These clathrin-containing complexes might be expected to comprise the soluble pool of clathrin that may represent both storage forms and intermediates in the cycle of disassembly and reassembly of the clathrin coated pit at the plasma membrane (Goud et al., 1985). However, in preliminary extraction experiments using the conditions described by Goud et al. (1985) to release the soluble form of clathrin, we found that while the percentage of clathrin released from cultured neurons was comparable to that reported for brain (Goud et al., 1985), the clathrin complex(es) remained insoluble (I. Pleasure, M. Black, and J. Keen, unpublished observations). If these clathrin-containing complexes participate in the cycle of coated membrane assembly and disassembly, then they represent novel intermediates in this cycle. However, most of the nonclathrin proteins of this complex do not correspond to identified components of the clathrin coat structure. These considerations suggest that the clathrin complexes may not represent intermediates in the coated membrane cycle, but rather may possess functions not commonly ascribed to clathrin. To begin defining these functions, it will be necessary to identify these other proteins and to learn more about the nature of the interactions that give rise to the complexes.

\section{References}

Ahle S, Mann A, Eichelsbacher U, Ungewickell E (1988) Structural relationships between clathrin assembly proteins from the Golgi and the plasma membrane. EMBO J 7:919-929.

Baas PW, Heidemann SR (1986) Microtubule reassembly from nucleating fragments during the regrowth of amputated neurites. J Cell Biol 103:917-927.

Baitinger C, Willard M (1987) Axonal transport of synapsin I-like proteins in rabbit retinal ganglion cells. J Neurosci 7:3723-3735.

Beckmann RP, Mizzen LA, Welch WJ (1990) Interaction of Hsp 70 with newly synthesized proteins: implications for protein folding and assembly. Science 248:850-854.

Black MM, Lasek RJ (1979) Axonal transport of actin: slow component $b$ is the principal source of actin for the axon. Brain Res 171: $401-413$.

Black MM, Lasek RJ (1980) Slow components of axonal transport: two cytoskeletal networks. J Cell Biol 86:616-623.

Bonner WM, Laskey RA (1974) A film detection method for tritiumlabeled proteins and nucleic acids in polyacrylamide gels. Eur J Biochem 46:83-88.

Brady ST (1984) Basic properties of fast axonal transport and the role of fast transport in axonal growth. In: Axonal transport in neuronal growth and regeneration (Elam JS, Cancalon P, eds), pp. 13-29. New York: Plenum.

Brady ST, Lasek RJ (1981) Nerve-specific enolase and creatine phosphokinase in axonal transport: soluble proteins and the axoplasmic matrix. Cell 23:515-523.

Brady ST, Tytell M, Heriot K, Lasek RJ (1981) Axonal transport of calmodulin: a physiologic approach to identification of long-term associations between proteins. J Cell Biol 89:607-614.

Bracll WA, Schlossman DM, Schmid SL, Rothman JE (1984) Dissociation of clathrin coats coupled to the hydrolysis of ATP: role of an uncoating ATPase. J Cell Biol 99:734-741.

Brodsky FM (1985) Clathrin structure characterized with monoclonal antibodies. II. Identification of in vivo forms of clathrin. J Cell Biol 101:2055-2062.
Chappell TG, Welch WJ, Schlossman DM, Palter KB, Schlesinger MJ, Rothman JE (1986) Uncoating ATPase is a member of the 70 kilodalton family of stress proteins. Cell 45:3-13.

Cheng TP, Byrd FI, Whitaker JN, Wood JG (1980) Immunocytochemical localization of coated vesicle protein in rodent nervous sys. tem. J Cell Biol 86:624-633.

Chestnut MH, Black MM, Keen JH (1986) Axonal transport of clathrin assembly polypeptides. J Cell Biol 103:54a.

Chirico WJ, Waters GM, Blobel G (1988) $70 \mathrm{~K}$ heat shock related proteins stimulate protein translocation into microsomes. Nature 332: $805-810$

Davis JQ, Bennett V (1985) Human erythrocyte clathrin and clathrinuncoating protein. J Biol Chem 260:14850-14856.

Davis JQ, Dansereau D, Johnstone RM, and Bennett V (1986) Selective externalization of an ATP-binding protein structurally related to the clathrin-uncoating ATPase/heat shock protein in vesicles containing terminal transferrin receptors during reticulocyte maturation. J Biol Chem 261:15368-15371.

de Waegh WS, Brady ST (1989) Axonal transport of a clathrin uncoating ATPase $(H S C 70)$ : a role for $H S C 70$ in the modulation of coated vesicle assembly in vivo. J Neurosci Res 23:433-440.

Deshaies RJ, Koch BD, Werner-Washburne M, Craig EA, Schekman, $R$ (1988) A subfamily of stress proteins facilitates translocation of secretory and mitochondrial precursor polypeptides. Nature 332:800805.

Flynn GC, Chappell TG, Rothman JE (1989) Peptide binding and release by proteins implicated as catalysts of protein assembly. Science 245:385-390.

Garner JA, Lasek RJ (1981) Clathrin is axonally transported as part of slow component $b$ : the microfilament complex. J Cell Biol 88:172178 .

Garner JA, Lasek RJ (1982) Cohesive axonal transport of the slow component b complex of polypeptides. J Neurosci 2:1824-1835.

Gething M-J, McCammon K, Sambrook J (1986) Expression of wildtype and mutant forms of influenza hemagglutinin: the role of folding in intracellular transport. Cell 46:939-950.

Giebel LB, Dworniczak BP, Bautz. EK (1988) Developmental regulation of a constitutively expressed mouse mRNA encoding a $72-\mathrm{kDa}$ heat shock-like protein. Dev Biol 125:200-207.

Goldstein JL, Brown MS, Anderson RGW, Russell DW, Schneider WJ (1985) Receptor-mediated endocytosis: concepts emerging from the LDL receptor system. Annu Rev Cell Biol 1:1-39.

Goud B, Huet C, Louvard D (1985) Assembled and unassembled pools of clathrin: a quantitative study using an enzyme immunoassay. J Cell Biol 100:521-527.

Gower DJ, Tytell M (1987) Axonal transport of clathrin-associated proteins. Brain Res 407:1-8.

Grafstein B, Forman DS (1980) Intracellular transport in neurons. Physiol Rev 60:1167-1283.

Greene LE, Eisenberg E (1990) Dissociation of clathrin from coated vesicles by the uncoating ATPase. J Biol Chem 265:6682-6687.

Hcuser JE, Reese TS (1973) Evidence for recycling of synaptic vesicle membrane during neurotransmitter release at the frog neuromuscular junction. J Cell Biol 57:315-344.

Heuser J, Steer CJ (1989) Trimeric binding of the $70-\mathrm{kD}$ uncoating ATPase to the vertices of clathrin triskelia: a candidate intermediate in the vesicle uncoating reaction. J Cell Biol 109:1457-1466.

Hurtley SM, Bole DG, Hoover-Litty H, Helenius A, Copeland CS (1989) Interactions of misfolded influenza virus hemagglutinin with binding protein (BiP). J Cell Biol 108:2117-2126.

Keen JH (1987) Clathrin assembly proteins: affinity purification and a model for coat assembly. J Cell Biol 105:1989-1998.

Keen JH (1990a) Clathrin and associated assembly and disassembly proteins. Ann Rev Biochem 59:415-438.

Keen JH (1990b) Clathrin assembly proteins and the organization of the coated membrane. Adv Cell Biol 3:153-176.

Keen JH, Black MM (1986) The phosphorylation of coated membrane proteins in intact neurons. J Cell Biol 102:1325-1333.

Keen JH, Willingham MC, Pastan IH (1981) Clathrin and coated vesicle proteins: immunological characterization. J Biol Chem 256: 2538-2544.

Kirchhausen T, Harrison SC (1984) Structural domains of clathrin heavy chains. J Cell Biol 99:1725-1734.

Lasek RJ, Garner JA, Brady ST (1984) Axonal transport of the cytoplasmic matrix. J Cell Biol 99:212s-221s. 
Lin CT, Garbern J, Wu J-Y (1982) Light and electron microscopic immunocytochemical localization of clathrin in rat cerebellum and kidney. J Histochem Cytochem 30:853-863.

Lisanti MP, Shapiro LS, Moskowitz N, Hua IL, Puszkin S, Schook, W (1982) Isolation and preliminary characterization of clathrin-associated proteins. Eur J Biochem 125:463-470.

Louvard D, Morris C, Warren G, Stanley K, Winkler F, Reggio H (1983) A monoclonal antibody to the heavy chain of clathrin. EMBO J 2:1655-1664.

Morre DM, Walter MM (1982) Coated microvesicles in central nervous system myelinated axons. Cell Biol Int Rep 6:401-405.

Oblinger MM, Foe LG, Kwiatkowska D, Kemp RG (1988) Phosphofructokinase in the rat nervous system: regional differences in activity and characteristics of axonal transport. J Neurosci Res 21: 25-34.

Patzer EJ, Schlossman DM, Rothman JE (1982) Release of clathrin from coated vesicles dependent upon a nucleoside triphosphate and a cytosol fraction. J Cell Biol 93:230-236.

Pelham HR (1989) Heat shock and the sorting of luminal ER proteins. EMBO J 8:3171-3176.

Peng I, Binder LI, Black MM (1985) Cultured neurons contain a variety of microtubule-associated proteins. Brain Res 361:200-211.

Perkins LA, Doctor JS, Zhang K, Stinson L, Pcrrimon N, Craig EA (1990) Molecular and developmental characterization of the heat shock cognate 4 gene of Drosophila melanogaster. Mol Cell Biol 10: 3232-3238.

Robinson MS (1987) 100-kD coated vesicle proteins: molecular heterogeneity and intracellular distribution studied with monoclonal antibodies. J Cell Biol 104:887-895.

Robinson MS, Pearse BM (1986) Immunofluorescent localization of $100 \mathrm{~K}$ coated vesicle proteins. J Cell Biol 102:48-54.

Rothman JE (1989) Polypeptide chain binding proteins: catalysts of protein folding and related processes in cells. Cell 59:591-601.

Rothman JE, Schmid SL (1986) Enzymatic recycling of clathrin from coated vesicles. Cell 46:5-9.
Schlossman DM, Schmid SL, Braell WA, Rothman JE (1984) An enzyme that removes clathrin coats: purification of an uncoating ATPase. J Cell Biol 99:723-733.

Schmid SL, Rothman JE (1985a) Enzymatic dissociation of clathrin cages in a two-stage process. J Biol Chem 260:10044-10049.

Schmid SL, Rothman JE (1985b) Two classes of binding sites for uncoating protein in clathrin triskelions. J Biol Chem 260:1005010056.

Schmid SL, Bracll WA, Rothman JE (1985) ATP catalyzes the sequestration of clathrin during enzymatic uncoating. J Biol Chem 260 10057-10062.

Stone GS, Hammerschlag R, Bobinski JA (1984) Involvement of coated vesicles in the initiation of fast axonal transport. Brain Res 291: 219-228.

Tytell M, Black MM, Garner JA, Lasek RJ (1981) Axonal transport: each major rate component reflects the movement of distinct macromolecular complexes. Science 214:179-181.

Ungewickell E (1985) The 70-kd mammalian heat shock proteins are structurally and functionally related to the uncoating protein that releases clathrin triskelia from coated vesicles. EMBO J 4(13A):33853391.

Ungewickell E, Branton D (1981) Assembly units of clathrin coats. Nature 289:420-422.

Welch WJ, Feramisco JR (1985) Rapid purification of mammalian 70,000-dalton stress proteins: affinity of the proteins for nucleotides. Mol Cell Biol 5:1229-1237.

Willard M (1977) The identification of two intra-axonally transported polypeptides resembling myocin in some respects in the rabbit visual system. J Cell Biol 75:1-11.

Zimmermann R, Sagstetter M, Lewis MJ, Pelham HR (1988) Seventykilodalton heat shock proteins and an additional component from reticulocyte lysate stimulate import of M13 procoat protein into microsomes. EMBO J 7:2875-2880. 